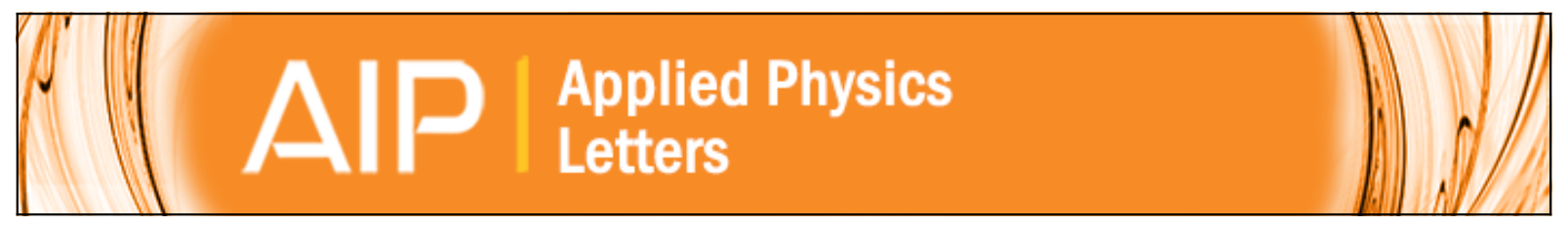

Local detection of enzymatic ion generation with polycrystalline silicon interdigitated electrodes and its application to biosensing

Roberto de la Rica, Antonio Baldi, and César Fernández-Sánchez

Citation: Applied Physics Letters 90, 074102 (2007); doi: 10.1063/1.2472718

View online: http://dx.doi.org/10.1063/1.2472718

View Table of Contents: http://scitation.aip.org/content/aip/journal/apl/90/7?ver=pdfcov

Published by the AIP Publishing
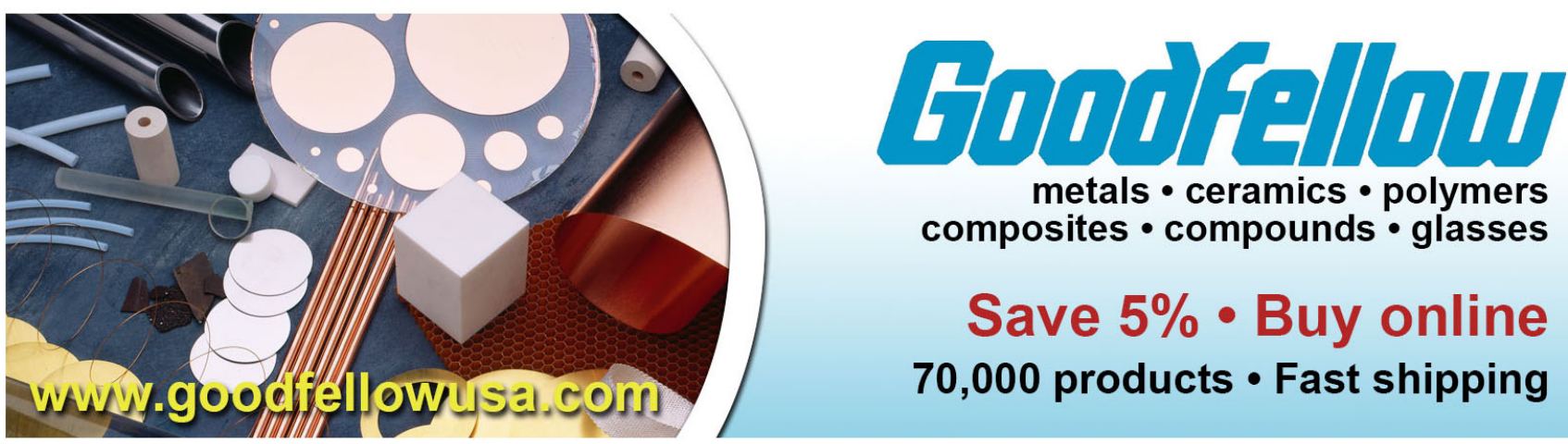

metals • ceramics $\cdot$ polymers composites $\cdot$ compounds $\cdot$ glasses

Save $5 \% \cdot$ Buy online 70,000 products $\cdot$ Fast shipping 


\title{
Local detection of enzymatic ion generation with polycrystalline silicon interdigitated electrodes and its application to biosensing
}

\author{
Roberto de la Rica, ${ }^{\text {a) }}$ Antonio Baldi, and César Fernández-Sánchez \\ Centro Nacional de Microelectrónica (IMB-CNM, CSIC), Esfera UAB, Campus UAB, 08193 Cerdanyola del \\ Vallés, Barcelona, Spain
}

(Received 28 December 2006; accepted 12 January 2007; published online 12 February 2007)

\begin{abstract}
Chips containing polycrystalline silicon interdigitated electrodes are modified with the enzyme urease. The sensors are able to detect changes in the resitivity of the solution near their surface, where the enzymatic reaction generates charged species. The electrodes are also grafted with an antigen and queried with different amounts of urease labeled antibody. The response of the modified electrodes is proportional to the amount of enzyme attached to the surface by the biorecognition event, thus validating the assay for biosensing applications. (c) 2007 American Institute of Physics.
\end{abstract}

[DOI: $10.1063 / 1.2472718$ ]

Patient-tailored prognosis, diagnosis, and therapy design are becoming achievable objectives due to the recent advances in genomics and proteomics. The chance of monitoring the state of a tissue, from detection of mutations in genomic deoxyribonucleic acid (DNA) to quantification of expressed ribonucleic acid levels and finding of proteins with an altered function, has opened a promising horizon in clinical biochemistry. Among the different approaches for biomolecule determination, biosensors offer the advantages of being robust, easy to handle, and capable of rendering information faster than other methodologies, which can only be performed in a laboratory by skilled staff. The signal generated by specific DNA-DNA or antigen-antibody interactions can be divided mainly in two groups. On the one hand, it may appear merely because of the presence of the analyte at the transducer surface. Examples of these so-called label-free biosensors are the detection of proteins by surface plasmon resonance, ${ }^{1,2}$ quarz crystal microbalance, ${ }^{3,4}$ or microcantilevers. ${ }^{5}$ On the other hand, the signal may be triggered by the presence of a label, usually conjugated to another biomolecule, e.g., a labeled antibody that recognizes its antigen attached to the sensor surface. Enzymes are one of the most commonly used labels in biosensing. They can be easily conjugated to biomolecules via different cross linkers and well known chemical procedures, and the reactions they catalyze can be detected either optically ${ }^{6,7}$ or electrochemically. ${ }^{8-11}$

In this letter, a biosensing principle is presented and tested. It consists in the generation of ions by an enzyme and the local detection of them by polycrystalline silicon interdigitated electrodes. The enzyme under study is urease, immobilized either via covalent linkage or by a biorecognition event at the transducer surface. In acidic media, the reaction catalyzed by urease can be described as

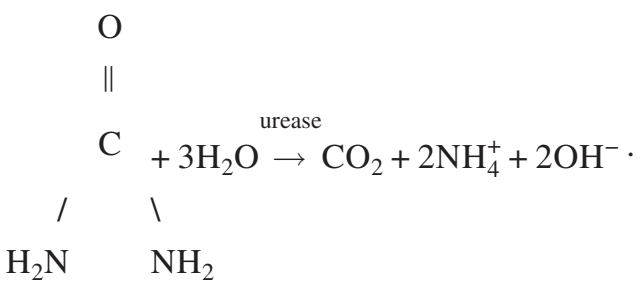

As a consequence, charged species appear at the electrodes surface. When the sensor is working under agitation, a stagnant layer a few micrometers thick is formed, where the arrival of substrate as well as the formation of products rapidly reaches a steady state. Thus, a concentration gradient of ions is created from the sensor surface to the bulk of the solution (Fig. 1). A suitable transducer, able to detect resistivity changes occurring close to its surface, should be very sensitive to the variations in the ion concentration created by the enzymatic reaction. Conversely, electrodes monitoring the resitivity of the bulk of the solution would measure a

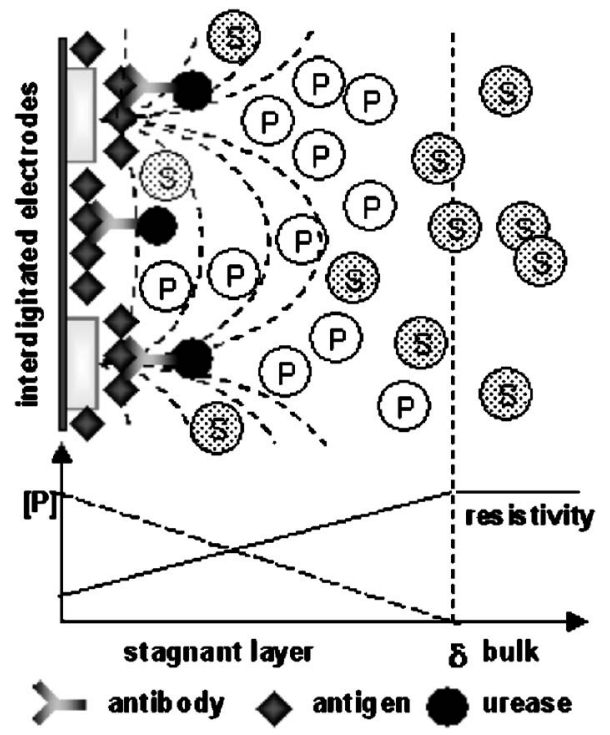

FIG. 1. Schematic representation of the bioassay. The biorecognition event drives urease to the electrodes surface, where the enzymatic reaction provokes the apparition of an ion concentration gradient. The geometry of the electrodes allows the local detection of the resultant resitivity change. 


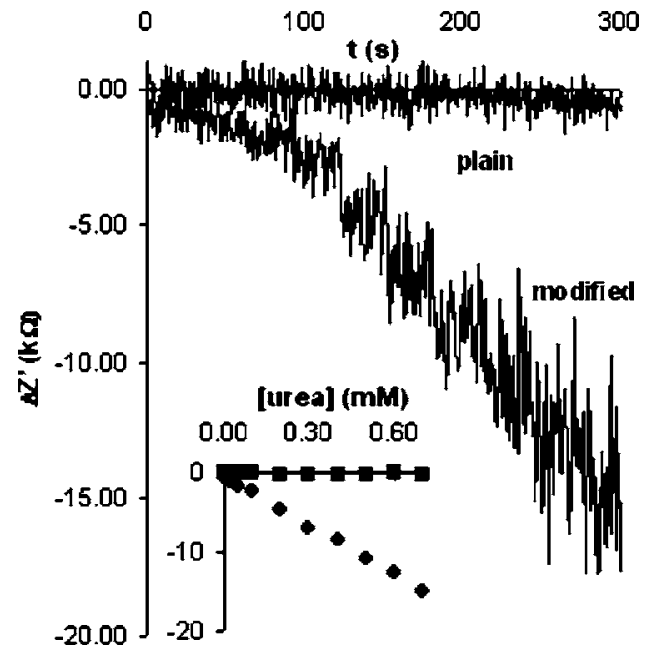

FIG. 2. $Z^{\prime}$ vs time plots for a plain and an urease modified sensor. Urea was added every $30 \mathrm{~s}$. The inset shows the variation of $Z^{\prime}$ with the substrate concentration for the same data set.

much lower change because in this region the concentration of added ions is minimum.

Here, planar interdigitated electrodes with $3 \mu \mathrm{m}$ width fingers are used as transducers. Recently, the capability of these electrodes to continuously monitor the resistivity of a solution by measuring the real part of the impedance at a given frequency was reported. ${ }^{12}$ They are made of heavily doped polycrystalline silicon, which is an excellent platform for biomolecule grafting through silanization. The short penetration depth of the electric fields makes them more sensitive to changes occurring in the region extending a few micrometers from their surface to the bulk of the solution. ${ }^{13}$ The capability of these electrodes to measure a resitivity decrease due to ion generation at their surface is evaluated with urease modified electrodes. Once cleaned with HF and 2.5\% $\mathrm{KOH}$, a chip was silanized with (3-aminopropyl)trimethoxysilane by putting it in a nitrogen purged chamber containing the silane overnight. The amino groups on the surface were turned to aldehyde ones by $1 \mathrm{~h}$ modification in a $12 \%$ glutaraldehyde solution in $0.1 M$ carbonate buffer. The modified electrodes were dipped in a $1 \mathrm{mg} / \mathrm{ml}$ urease solution containing $50 \mathrm{mM}$ sodium cyanoborohydride at $4{ }^{\circ} \mathrm{C}$ overnight. After a cleaning step in a $0.1 \%$ Tween 20 solution, the treatment rendered the sensor modified with covalently attached urease at its surface. Three experiments were then performed. In the first experiment the biosensor was immersed in a $50 \mathrm{mM}$ glycine solution $p \mathrm{H}$ of 5.7 , and the formation of charged species at its surface was monitored by measuring the real part of the impedance $\left(Z^{\prime}\right)$ while adding the substrate at fixed time intervals $(30 \mathrm{~s})$. In the second experiment, two pairs of electrodes were placed in the reaction chamber, one of them was modified with urease and the other one was not. The electrodes recording the impedance were the plain ones, while the enzyme electrodes only contribute charged species to the bulk of the solution due to the catalytic reaction taking place at their surface. In this case, the ion generation is detached from the detection device and the monitored resistivity changes take place in the bulk of the solution. Finally, the experiment was repeated with a plain sensor alone, in order to monitor other variations not due to the enzymatic reaction. Results from this experiment were subtracted from the two former ones. Figure 2 shows

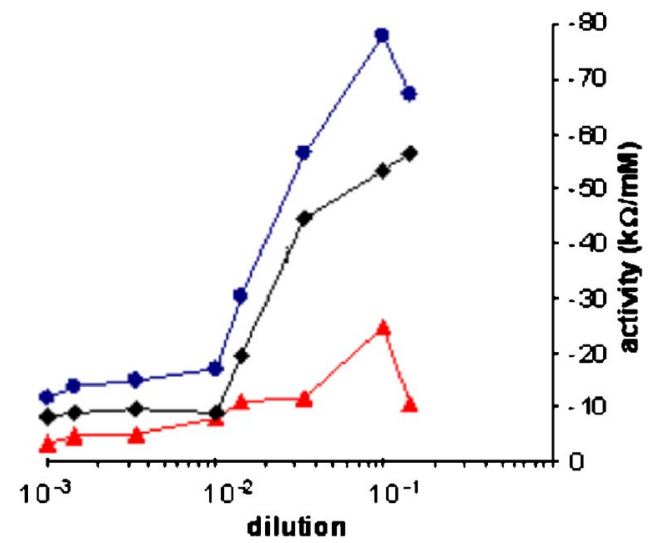

FIG. 3. (Color online) Subtraction of the response of antigen-grafted electrodes (circles) from the blank (triangle) yields an specific response curve (squares). The plot shows a correlation between the amount of urease attached to the surface (dilution) and the response of the electrode to urea.

the raw data of $Z^{\prime}$ versus time curve for the biosensor and the plain sensor. When the enzymatic reaction takes place at the electrodes, the resulting ion generation is detected and the relationship between the impedance and the urea concentration is linear in a certain range. Conversely, when the reaction takes place in the bulk of the solution, no impedance change is observed. This experiment undoubtedly demonstrates that the interdigitated electrodes used in these experiments are able to locally monitor the enzymatic production of charged species, and that this mode of detection is more sensitive to ion generation than bulk detection. Moreover, these biosensors are virtually independent of the chamber volume where the enzymatic reaction takes place, and therefore could be easily integrated in a microfluidic device.

Once the transduction principle is demonstrated, the feasibility of the proposed assay for biosensing is tested. The reaction between rabbit IgG (antigen) and urease labeled anti rabbit $\operatorname{IgG}$ (antibody) is used as the biorecognition event. The conjugation of the enzyme to the antibody has been reported elsewhere. ${ }^{14}$ Rabbit $\mathrm{IgG}$ was grafted to the chip surface as before. The sensors were blocked in $100 \mathrm{mM}$ tris(hydroxymethyl)aminomethane (TRIS) buffer, $p \mathrm{H}$ of 8.6 containing $1 \%$ albumin from bovine serum, and $50 \mathrm{mM}$ ethanolamine at $4{ }^{\circ} \mathrm{C}$ overnight. Dilutions from 1:7 to $1: 10^{3}$ of urease labeled antibody were prepared in $100 \mathrm{mM}$ TRIS buffer $p \mathrm{H}$ of 7 containing $10 \mathrm{mM}$ thioacetamide, $10 \mathrm{mM}$ Ethylenediaminetetraacetic acid, and $140 \mathrm{mM} \mathrm{NaCl}$. Antigen modified electrodes were dipped for $1 \mathrm{~h}$ in these solutions and, as a result of the biorecognition event, the electrodes were coated with different amounts of enzyme. Upon cleaning in $0.1 \%$ Tween 20 , the biosensors were calibrated with urea as decribed above, and the slope of the linear part of the curve $Z^{\prime}$ versus concentration taken as a measure of the activity of the enzyme electrode. A blank curve was also obtained by interrogating anti rabbit IgG grafted electrodes with urease labeled anti rabbit IgG. Subtraction of the antigen curve to the blank curve rendered the plot corresponding to the specific interaction. When the activity is plotted versus the antibody dilution in semilogarithmic scale, a typical sigmoidal curve with a linear range is obtained (Fig. 3). These experiments confirm that the proposed biosensing principle can be applied to detect and quantify proteins. These results, together with the ones shown above, and the fact that the electrodes are miniaturized and made with well known pro- 
cesses in the microelectronics industry, make the biosensors good candidates for the fabrication of integrated protein arrays.

${ }^{1}$ R. Kurita, Y. Yokota, Y. Sato, F. Mizutani, and O. Niwa, Anal. Chem. 78, 5525 (2006).

${ }^{2}$ N. Kanoh, M. Kyo, K. Inamori, A. Ando, A. Asami, A. Nakao, and H. Osada, Anal. Chem. 78, 2226 (2006).

${ }^{3}$ A. E. Gerdon, D. W. Wright, and D. E. Cliffel, Anal. Chem. 77, 304 (2005).

${ }^{4}$ H. Zeng, H. Wang, F. Chen, H. Xin, G. Wang, L. Xiao, K. Song, D. Wu, Q. He, and G. Shen, Anal. Biochem. 351, 69 (2006).

${ }^{5}$ G. Wu, R. H. Datar, K. M. Hansen, T. Thundat, R. J. Cote, and A. Majumdar, Nat. Biotechnol. 19, 856 (2001).

${ }^{6}$ T. Konry, A. Novoa, Y. Shemer-Avni, N. Hanuka, S. Cosnier, A. Lepellec, and R.S. Marks, Talanta 77, 1771 (2005).

${ }^{7}$ W. L. Xing, L. R. Ma, Z. H. Jiang, F. H. Cao, and M. H. Jia, Talanta 52, 879 (2000).

${ }^{8}$ J. Albers, T. Grinwald, E. Nebling, G. Piechotta, and R. Hintsche, Anal. Bioanal. Chem. 377, 521 (2003).

${ }^{9}$ C. Fernández-Sánchez, M. B. González-García, and A. Costa-García, Biosens. Bioelectron. 14, 917 (2000).

${ }^{10}$ M. S. Wilson and W. Nie, Anal. Chem. 78, 6476 (2006).

${ }^{11}$ C. Fernández-Sánchez, C. J. McNeil, K. Rawson, and O. Nilsson, Anal. Chem. 76, 5649 (2004)

${ }^{12}$ R. de la Rica, C. Fernández-Sánchez, and A. Baldi, Electrochem. Commun. 8, 1239 (2006).

${ }^{13}$ N. F. Sheppard, D. J. Mears, and A. Guiseppi-Elie, Biosens. Bioelectron. 11, 967 (1996).

${ }^{14}$ T. J. Hermanson,Bioconjugate Techniques (Academic, Elsevier, 1996), p. 173. 